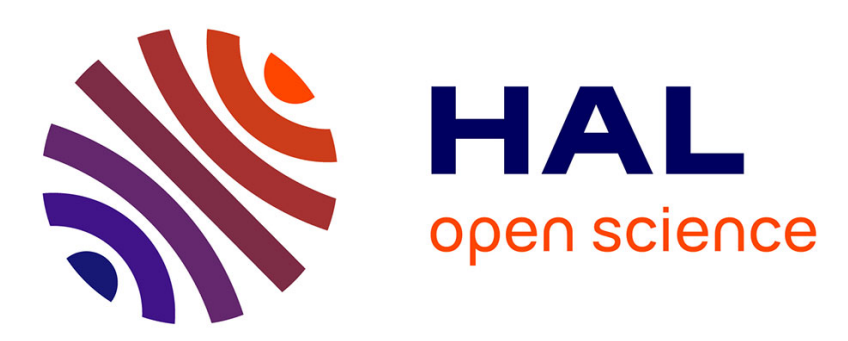

\title{
An Analysis of Sum-Based Incommensurable Belief Base Merging
}

Salem Benferhat, Sylvain Lagrue, Julien Rossit

\section{To cite this version:}

Salem Benferhat, Sylvain Lagrue, Julien Rossit. An Analysis of Sum-Based Incommensurable Belief Base Merging. hird International Conference on Scalable Uncertainty Management (SUM'09), 2009, Washington, United States. pp.55-67. hal-00867746

\section{HAL Id: hal-00867746 https://hal.science/hal-00867746}

Submitted on 30 Sep 2013

HAL is a multi-disciplinary open access archive for the deposit and dissemination of scientific research documents, whether they are published or not. The documents may come from teaching and research institutions in France or abroad, or from public or private research centers.
L'archive ouverte pluridisciplinaire HAL, est destinée au dépôt et à la diffusion de documents scientifiques de niveau recherche, publiés ou non, émanant des établissements d'enseignement et de recherche français ou étrangers, des laboratoires publics ou privés. 


\title{
An Analysis of Sum-Based Incommensurable Belief Base Merging
}

\author{
Salem Benferhat, Sylvain Lagrue, and Julien Rossit \\ CRIL-CNRS UMR 8081, \\ Université d'Artois, Faculté des Sciences Jean Perrin \\ rue Jean Souvraz, SP18. 62307 Lens France \\ \{benferhat, lagrue,rossit\}@cril.univ-artois.fr
}

\begin{abstract}
Different methods have been proposed for merging multiple and potentially conflicting informations. Sum-based operators offer a natural method for merging commensurable prioritized belief bases. Their popularity is due to the fact that they satisfy the majority property and they adopt a non cautious attitude in deriving plausible conclusions.

This paper analyses the sum-based merging operator when sources to merge are incommensurable, namely they do not share the same meaning of uncertainty scales. We first show that the obtained merging operator can be equivalently characterized either in terms of an infinite set of compatible scales, or by a well-known Pareto ordering on a set of models. We then study different families of compatible scales useful for merging process. This paper also provides a postulates-based analysis of our merging operators.
\end{abstract}

\section{Introduction}

The problem of merging multiple-source information is crucial for many applications. Indeed, many situations require to take into account several potentially conflicting pieces of information, such as distributed databases frameworks, multi-agent systems, or distributed information in general (e.g. semantic web). This kind of situation leads to perform some combination operations on available pieces of information, which is well known as a data fusion problem.

Different merging operators have been proposed in the literature to merge prioritized pieces of information issued from different sources [1-3]. Most of existing merging methods assume that ranks associated with beliefs are commensurable from one source to another. This commensurability assumption may make sense in some situations, when it is possible to obtain a reference scale between sources. However, it can appear to be too strong for other applications. Only few works have addressed the issue of merging incommensurable ranked belief bases $[4,5]$.

In this paper, we first provide, in Section 3, a natural extension of the sumbased fusion mode to deal with incommensurable belief bases. This extension uses the concept of compatible scales to define the result of merging. We show, in the same section, that the fusion can also be characterized in terms of a Pareto-ordering on possible worlds. We then analyze, in Section 4, the logical 
behavior of the sum-based merging operator in commensurable and incommensurable cases. As a surprising result, the majority postulate is no longer valid when dealing with incommensurable belief bases. Even worse, the sum-based merging operator become majority-independent. Section 5 presents several inference relations based on some selection functions of compatible scales (such as the ones obtained from linear transformations or bounded compatible scales). In particular, we analyze the impact of these selection functions on the satisfaction of rationality postulates, and on the prudence of merging operators. Section 6 provides a brief comparison with some other merging approaches.

Before developing in details these results, we first provide some backgrounds on ranked belief bases and sum-based merging of commensurable belief bases.

\section{Merging Ranked Belief Bases}

Let $\mathcal{L}$ be a finite propositional language. We denote by $\Omega$ the set of interpretations of $\mathcal{L}$ and by $\omega$ an element of $\Omega$. Greek letters $\varphi$ and $\psi$ denote propositional formulas. $\equiv$ denotes a logical equivalence, $\operatorname{Mod}(\varphi)$ represents the set of models of $\varphi, \top$ and $\perp$ represent respectively a tautology and a contradiction.

\subsection{Ranked Belief Bases}

A ranked belief base is a multi-set of ranked formulas. It contains beliefs provided by a given source. The term belief is used when pieces of information provided by sources are uncertain. A ranked belief is represented by a propositional formula associated with a rank. This rank represents the amount of uncertainty associated with the formula, and can simply expresses the reliability of the source which provides this belief. A ranked belief base is a convenient framework to represent uncertain (or prioritized) pieces of information:

Definition 1 (Ranked belief base). A ranked belief base $B_{i}$ is a multi-set of ranked propositional formulas $B_{i}=\left\{\left(\varphi_{i j}, R_{B_{i}}\left(\varphi_{i j}\right)\right), j \in\left\{1, \ldots, m_{i}\right\}\right\}$, where $\varphi_{i j} \in \mathcal{L}$, and $R_{B_{i}}\left(\varphi_{i j}\right) \in \mathbb{N}^{*}$.

$\left(\varphi_{i j}, R_{B_{i}}\left(\varphi_{i j}\right)\right)$ means that $\varphi_{i j}$ has a priority rank of at least $R_{B_{i}}\left(\varphi_{i j}\right)$. Intuitively, formulas associated with highest ranks are those which are preferred for a given source (or agent). Only strictly positive ranks are represented. Ranked belief bases are used in different frameworks, such as possibility theory [6] or ordinal conditional functions (OCF) [7-9]. We denote by $B_{i}^{*}$ the set of propositional formulas obtained from $B_{i}$ by ignoring ranks associated with formulas.

Given a ranked belief base, a total pre-order on interpretations of $\Omega$ can be derived as follows: $\omega$ is preferred to $\omega^{\prime}$ if and only if the strongest belief falsified by $\omega$ is less important than the strongest belief falsified by $\omega^{\prime}$. More precisely:

Definition 2 ( $\kappa$-functions). A ranking function $\kappa_{B_{i}}$ associated with a ranked belief base $B_{i}$ is a function that maps each interpretation $\omega \in \Omega$ to an integer $\kappa_{B_{i}}(\omega)$ such that:

$$
\kappa_{B}(\omega)= \begin{cases}0 & \text { if } \forall\left(\varphi_{i j}, R_{B_{i}}\left(\varphi_{i j}\right)\right) \in B_{i}, \omega \models \varphi_{i j} \\ \max \left\{R_{B_{i}}\left(\varphi_{i j}\right): \omega \not \varphi_{i j},\left(\varphi_{i j}, R_{B_{i}}\left(\varphi_{i j}\right)\right) \in B_{i}\right\} & \text { otherwise. }\end{cases}
$$


Interpretations associated with lower ranks represent agent's current beliefs. This ordering is the basis of possibilistic logic [6] and adjustment revision [8].

Example 1. Let us consider the ranked belief base $B=\{(\neg a \vee b, 8),(a \vee b, 5),(a, 2)\}$. Table 1 gives the $\kappa$-function $\kappa_{B}$ associated with $B . \omega_{3}$ is the preferred interpretation and will represent agent's current beliefs.

\begin{tabular}{c|c|c|c}
\hline$\omega_{i} \in \Omega$ & $\mathrm{a}$ & $\mathrm{b}$ & $\kappa_{B}\left(\omega_{i}\right)$ \\
\hline$\omega_{0}$ & 0 & 0 & 5 \\
$\omega_{1}$ & 0 & 1 & 2 \\
$\omega_{2}$ & 1 & 0 & 8 \\
$\omega_{3}$ & 1 & 1 & $\mathbf{0}$ \\
\hline
\end{tabular}

Table 1. An example of $\kappa$-function

It is important to note that beliefs are inserted as they are and as they come from their sources, and we do not add derived beliefs. This is the spirit of what is called "belief bases" by Nebel [10]. Hence, the same belief can be present several times in $B_{i}$ and this explains why we consider it as a multi-set. Equivalent beliefs have different identification (the identification can be an arbitrary numbering of the beliefs in $B_{i}$ ). We do not make these identifications explicit since it renders the notation heavy. Formulas of belief bases are distinguished from plausible conclusions which are derived from beliefs.

\subsection{Sum-Based Fusion of Commensurable Bases}

This section recalls a sum-based merging method. Let $E=\left\{B_{1}, \ldots, B_{n}\right\}$ be a multi-set of $n$ ranked belief bases issued from $n$ sources, and let $\mu$ be a propositional formula representing integrity constraints to satisfy. The aim of merging is, given $E$ and $\mu$, to rank-order different interpretations of $\Omega$ with respect to pieces of information provided by sources. This ordering is often obtained using a merging operator denoted here by $\triangle^{\mu}$. Given $E, \triangle^{\mu}$, we denote by $\triangleleft^{E}$ the ordering on $\Omega$ induced by $\triangle^{\mu}$ and $E$. We denote by $\triangle^{\mu}(E)$ the so-called belief set which represents the set of actual beliefs obtained after merging $E$ and $\mu$ by $\triangle^{\mu} . \triangle^{\mu}(E)$ is defined as usually, namely it is such that its models are those which are minimal with respect to $\triangleleft^{E}$. In the literature, different methods for merging $E$ have been proposed (e.g. [2,3,11]). This paper focuses on a sum-based fusion, denoted by $\triangle_{\Sigma}^{\mu}$.

To compute the result of merging, each interpretation $\omega$ is associated with a profile, denoted by $\nu_{E}(\omega)$, and defined by:

$$
\nu_{E}(\omega)=\left\langle\kappa_{B_{1}}(\omega), \ldots, \kappa_{B_{n}}(\omega)\right\rangle
$$

It represents the consistency degree of an interpretation $\omega$ with respect to ranked bases to merge. The computation of $\triangle_{\Sigma}^{\mu}$ is achieved in two steps: first combine the consistency degrees $\kappa_{B_{i}}(\omega)$ 's with the Sum operator $\Sigma$, and then rank-order interpretations with respect to their obtained ranks. More formally: 
Definition $3\left(\triangleleft_{\Sigma}^{E}\right)$. Let $\omega$ and $\omega^{\prime}$ be two interpretations of $\Omega$, and $\nu_{E}(\omega)$, $\nu_{E}\left(\omega^{\prime}\right)$ be their respective profiles. Then $: \omega \triangleleft_{\Sigma}^{E} \omega^{\prime}$ iff $\Sigma\left(\nu_{E}(\omega)\right)<\Sigma\left(\nu_{E}\left(\omega^{\prime}\right)\right)$, where $\Sigma\left(\nu_{E}(\omega)\right)=\sum_{i=1, . ., n} \kappa_{B_{i}}(\omega)$.

Models of $\triangle_{\Sigma}^{\mu}(E)$ are models of $\mu$ which are minimal with respect to $\triangleleft_{\Sigma}^{E}$ :

$$
\operatorname{Mod}\left(\triangle_{\Sigma}^{\mu}(E)\right)=\operatorname{Min}\left(\operatorname{Mod}(\mu), \triangleleft_{\Sigma}^{E}\right)
$$

The sum-based merging operator is majority dependent : the repetition of a same piece of information may affect the result of merging. This kind of operator is particularly well adapted if sources (or agents) are assumed to be independent.

Example 2. Let us consider $E=\left\{B_{1}, B_{2} B_{3}\right\}$ where $B_{1}=\{(a, 6),(b, 3)\}, B_{2}=$ $\{(a \vee b, 3),(\neg b, 1)\}$ and $B_{3}=\{(\neg a, 5\}$. Assume that $\mu \equiv \neg a \vee \neg b$. Profiles associated with interpretations are given by Table 2 . We have $\operatorname{Mod}\left(\triangle_{\Sigma}^{\mu}(E)\right)=\left\{\omega_{1}\right\}$.

\begin{tabular}{c|c|c|c|c}
\hline$\omega \in \Omega$ & $\mathrm{b}$ & $\nu_{E}(\omega)$ & $\Sigma\left(\nu_{E}(\omega)\right)$ \\
\hline$\omega_{0}$ & 0 & 0 & $\langle 6,3,0\rangle$ & 9 \\
$\omega_{1}$ & $\mathbf{0}$ & $\mathbf{1}$ & $\langle\mathbf{6 , 1 , 0}\rangle$ & $\mathbf{7}$ \\
$\omega_{2}$ & 1 & 0 & $\langle 3,0,5\rangle$ & 8 \\
$\omega_{3}$ & 1 & 1 & $\langle 0,1,5\rangle$ & 6 \\
\hline
\end{tabular}

Table 2. Profiles associated with interpretations

\section{Extension of the Sum-Based Operator for Merging Incommensurable Belief Bases}

\subsection{Compatible Scales Merging Approach}

The sum-based merging operator defined above assumes that ranks, associated with formulas, have to be commensurable in order to sum them. Such assumption can be too strong for some applications, for instance when information is obtained from sources with unknown quality (e.g. web). A natural way to merge incommensurable belief bases consists in using possible common scales, called compatible scales [5]. A compatible scale affects new ranks to beliefs such that initial relative orders between beliefs of each agent are preserved.

Definition 4 (Compatible scale). A compatible scale $\mathcal{S}$ is a function that maps $E=\left\{B_{1}, \ldots, B_{n}\right\}$ to $E^{\mathcal{S}}=\left\{B_{1}^{\mathcal{S}}, \ldots, B_{n}^{\mathcal{S}}\right\}$ such that for all $B_{i} \in E$ :

(i) $B_{i}^{\mathcal{S}}=\left\{\left(\varphi_{i j}, \mathcal{S}\left(\varphi_{i j}\right)\right):\left(\varphi_{i j}, R_{B_{i}}\left(\varphi_{i j}\right)\right) \in B_{i}\right\}$

(ii) $\forall B_{i} \in E, \forall\left(\varphi_{i j}, R_{B_{i}}\left(\varphi_{i j}\right)\right) \in B_{i}, \forall\left(\varphi_{i j^{\prime}}, R_{B_{i}}\left(\varphi_{i j^{\prime}}\right)\right) \in B_{i}$, $R_{B_{i}}\left(\varphi_{i j}\right) \leq R_{B_{i}}\left(\varphi_{i j^{\prime}}\right)$ iff $\mathcal{S}\left(\varphi_{i j}\right) \leq \mathcal{S}\left(\varphi_{i j^{\prime}}\right)$.

The following example shows that compatible scales are not unique. 


\begin{tabular}{c|c|c|ccc}
\hline & $\varphi_{i j}$ & $R_{B_{i}}\left(\varphi_{i j}\right)$ & $\mathcal{S}_{1}\left(\varphi_{i j}\right)$ & $\mathcal{S}_{2}\left(\varphi_{i j}\right)$ & $\mathcal{S}_{3}\left(\varphi_{i j}\right)$ \\
\hline$B_{1}$ & $a$ & 6 & 2 & 6 & 1 \\
& $b$ & 3 & 1 & 4 & 5 \\
\hline$B_{2}$ & $a \vee b$ & 3 & 2 & 3 & 3 \\
& $\neg b$ & 1 & 1 & 2 & 1 \\
\hline$B_{3}$ & $\neg a$ & 5 & 1 & 4 & 5 \\
\hline
\end{tabular}

Table 3. Examples of compatible scales

Example 3. Let us consider $E=\left\{B_{1}, B_{2}, B_{3}\right\}$ be belief bases provided by Example 2. Table 3 gives three possible scales: $\mathcal{S}_{1}, \mathcal{S}_{2}$ and $\mathcal{S}_{3}$. Scales $\mathcal{S}_{1}$ and $\mathcal{S}_{2}$ are compatible, because they preserve initial orders induced by each base. However, $\mathcal{S}_{3}$ is not a compatible one: it inverses priority between beliefs of $B_{1}$.

As we already pointed out, beliefs are considered as self justified. A compatible scale does not directly handle beliefs but rather normalize ranks associated with beliefs. If, for instance, one have two bases: $B_{1}=\{(a, 1),(b, 2)\}$, and $B_{2}=\{(b, 3),(a, 5)\} ;$ a possible common scale is $\mathcal{S}$ such that $B_{1}^{\mathcal{S}}=\{(a, 2),(b, 3)\}$, and $B_{2}^{\mathcal{S}}=\{(b, 2),(a, 3)\}$. This scale is compatible since it simply preserves the initial ordering between beliefs of $B_{1}$ and between beliefs of $B_{2}$.

The set of scales compatible with $E$ is denoted by $\mathbb{S}_{E}$. Note that $\mathbb{S}_{E}$ is never empty (it is enough to consider a scale that simply uses initial ranks, which is trivially compatible). Given a compatible scale $\mathcal{S} \in \mathbb{S}_{E}$, we denote by $B_{i}^{\mathcal{S}}$ the belief base obtained from $B_{i}$ by using a compatible scale $\mathcal{S}$. More formally, $B_{i}^{\mathcal{S}}$ is obtained by replacing each pair $\left(\varphi_{i j}, R_{B_{i}}\left(\varphi_{i j}\right)\right)$ by $\left(\varphi_{i j}, \mathcal{S}\left(\varphi_{i j}\right)\right)$. Moreover, we denote by $E^{\mathcal{S}}$ the multi-set obtained by application of $\mathcal{S}$ on each $B_{i}$ from $E$.

Definition 5 (Definition of $\triangleleft_{\Sigma}^{E}$ ). Let $\omega, \omega^{\prime}$ be two interpretations of $\Omega$. Then:

$$
\omega \triangleleft_{\Sigma}^{E} \omega^{\prime} \quad \text { iff } \forall \mathcal{S} \in \mathbb{S}_{E}, \omega \triangleleft_{\Sigma}^{E^{\mathcal{S}}} \omega^{\prime}
$$

where $\triangleleft \frac{E_{\Sigma}^{\mathcal{S}}}{\text { is }}$ the result of applying the Definition 3 on $E^{\mathcal{S}}$.

Models of $\boldsymbol{\Lambda}_{\Sigma}^{\mu}(E)$ are again: $\operatorname{Mod}\left(\boldsymbol{\Lambda}_{\Sigma}^{\mu}(E)\right)=\operatorname{Min}\left(\operatorname{Mod}(\mu), \boldsymbol{\iota}_{\Sigma}^{E}\right)$.

Example 4. Let consider again $B_{1}=\{(a, 6),(b, 3)\}, B_{2}=\{(a \vee b, 3),(\neg b, 1)\}$ and $B_{3}=\{(\neg a, 5\}$. Table 4 provides profiles associated with interpretations for each of two compatible scales, $\mathcal{S}_{1}$ and $\mathcal{S}_{2}$. Bold elements represent models of $\triangle_{\Sigma}^{\mu}\left(E^{\mathcal{S}_{i}}\right)$. For instance, $\omega_{2}$, and $\omega_{3}$ are models of $\triangle_{\Sigma}^{\mu}\left(E^{\mathcal{S}_{1}}\right)$ according to $\mathcal{S}_{1}$.

\begin{tabular}{|c|c|c|c|c|c|}
\hline $\bar{\omega} \in \Omega$ & $a b$ & $\nu_{E} \mathcal{S}_{1}(\omega)$ & $\Sigma_{\mathcal{S}_{1}}$ & $\nu_{E} \mathcal{S}_{2}(\omega)$ & $\Sigma_{\mathcal{S}_{2}}$ \\
\hline$\omega_{0}$ & \begin{tabular}{||ll||l|l|l} 
& 0
\end{tabular} & $\langle 2,2,0\rangle$ & 4 & $\langle 6,3,0\rangle$ & 9 \\
\hline$\omega_{1}$ & \begin{tabular}{|ll|l}
0 & 1
\end{tabular} \mid & $\mid\langle 2,1,0\rangle$ & 3 & $\langle 6,2,0\rangle$ & 8 \\
\hline$\omega_{2}$ & \begin{tabular}{|lll|l}
1 & 0
\end{tabular} & $\langle\mathbf{1}, \mathbf{0}, \mathbf{1}\rangle$ & 2 & $\langle 4,0,4\rangle$ & 8 \\
\hline$\omega_{3}$ & \begin{tabular}{|lll|l}
1 & 1
\end{tabular} & $\mid\langle\mathbf{0}, \mathbf{1}, \mathbf{1}\rangle$ & 2 & $\langle 0,2,4\rangle$ & 6 \\
\hline
\end{tabular}

Table 4. Profiles associated with interpretations 


\subsection{Characterization of the Result of Merging}

This subsection shows that it is possible to characterize the result of fusion without comparing all compatible scales. The following proposition shows that an interpretation $\omega$ is a model of $\boldsymbol{\Lambda}_{\Sigma}^{\mu}(E)$ if and only if there exists a compatible scale $\mathcal{S}$ where $\omega$ is a model of $\triangle_{\Sigma}^{\mu}\left(E^{\mathcal{S}}\right)$. More formally:

Proposition 1. Let $E$ be a multi-set of ranked belief bases. Then $\omega \in \operatorname{Mod}\left(\boldsymbol{\Lambda}_{\Sigma}^{\mu}(E)\right)$, if and only if there exists a compatible scaling $\mathcal{S}$ such that $\omega \in \operatorname{Mod}\left(\triangle_{\Sigma}^{\mu}\left(E^{\mathcal{S}}\right)\right)$.

We now generalize this proposition by characterizing the whole ordering $\varangle_{\Sigma}^{E}$ and not only its minimal elements. It turns out that $\varangle_{\Sigma}^{E}$ corresponds to the well-known Pareto Criterion. Namely:

Proposition 2. Let $\omega$ and $\omega^{\prime}$ be two interpretations of $\Omega$. Then $\omega \mathbf{4}_{\Sigma}^{E} \omega^{\prime}$ iff: (i) $\forall j \in\{1, . ., n\}, \kappa_{B_{j}}(\omega) \leq \kappa_{B_{j}}\left(\omega^{\prime}\right)$ and (ii) $\exists i \in\{1, \ldots, n\}, \kappa_{B_{i}}(\omega)<\kappa_{B_{i}}\left(\omega^{\prime}\right)$.

The first condition means that $\omega$ is at least as preferred as $\omega^{\prime}$ with respect to each belief base, while the second condition means that at least one base expresses a strict preference for $\omega$.

\section{Logical Behavior and Rational Postulates}

Many postulates have been proposed in the literature to characterize merging operators under constraints (see [3] for details). These postulates are defined when belief bases are represented by propositional formulas. In our framework, these postulates have been adapted (see [5] for details) as follows:

(IC0) $\triangle^{\mu}(E) \models \mu$;

(IC1) If $\mu$ is consistent, then $\triangle^{\mu}(E)$ is consistent;

(IC2 $^{*}$ ) If $\bigwedge_{B \in E} B^{*}$ is consistent with $\mu$, then $\triangle^{\mu}(E) \equiv \bigwedge_{B \in E} B^{*} \wedge \mu$

$\left(\right.$ IC3 $\left.^{*}\right)$ If $E_{1} \equiv_{R} E_{2}$ and $\mu_{1} \equiv \mu_{2}$, then $\triangle^{\mu_{1}}\left(E_{1}\right) \equiv \triangle^{\mu_{2}}\left(E_{2}\right)$

(IC4 $\left.{ }^{*}\right)$ If $B_{1}^{*} \models \mu$ and $B_{2}^{*} \models \mu$, then $\triangle^{\mu}\left(\left\{B_{1}, B_{2}\right\}\right) \wedge B_{1}^{*}$ is consistent iff $\triangle^{\mu}\left(\left\{B_{1}, B_{2}\right\}\right) \wedge B_{2}^{*}$ is consistent;

(IC5) $\triangle^{\mu}\left(E_{1}\right) \wedge \triangle^{\mu}\left(E_{2}\right) \models \triangle^{\mu}\left(E_{1} \sqcup E_{2}\right)$;

(IC6) If $\triangle^{\mu}\left(E_{1}\right) \wedge \triangle^{\mu}\left(E_{2}\right)$ is consistent, then $\triangle^{\mu}\left(E_{1} \sqcup E_{2}\right) \models \triangle^{\mu}\left(E_{1}\right) \wedge \triangle^{\mu}\left(E_{2}\right) ;$

(IC7) $\triangle^{\mu_{1}}(E) \wedge \mu_{2} \models \triangle^{\mu_{1} \wedge \mu_{2}}(E)$;

(IC8) If $\triangle^{\mu_{1}}(E) \wedge \mu_{2}$ is consistent, then $\triangle^{\mu_{1} \wedge \mu_{2}}(E) \models \triangle^{\mu_{1}}(E) \wedge \mu_{2}$.

Additional postulates have been proposed in [3]:

(IC6') If $\triangle^{\mu}\left(E_{1}\right) \wedge \triangle^{\mu}\left(E_{2}\right)$ is consistent, then $\triangle^{\mu}\left(E_{1} \bigsqcup E_{2}\right) \models \triangle^{\mu}\left(E_{1}\right) \vee \triangle^{\mu}\left(E_{2}\right)$;

(MAJ) $\exists m \in \mathbb{N}: \triangle^{\mu}\left(E \sqcup B_{i}^{m}\right) \models B_{i}^{*}$, (with $B_{i}^{m}=\left\{B_{i}\right\} \sqcup \ldots \sqcup\left\{B_{i}\right\} m$ times and $\sqcup$ the multi-set union) ; 
(MI) $\forall m, \triangle^{\mu}\left(E_{1} \sqcup E_{2}^{m}\right) \equiv \triangle^{\mu}\left(E_{1} \sqcup E_{2}\right)$.

The majority postulate (MAJ) characterizes majoritarian merging operators: if a given set of beliefs is repeated often enough, this set of beliefs should be accepted in the result of merging. At the opposite, the majority independence postulate states that the result of merging is independent of the repetition of beliefs. (IC6') is a weakened version of (IC6). We also introduce in this paper a stronger version of $\left(\mathbf{I C} 4^{*}\right)$, called the consensus postulate:

(CSS) $\forall B_{i} \in E$, if $B_{i} \models \mu$, then $B_{i}^{*} \wedge \triangle^{\mu}(E)$ is consistent.

\subsection{The Commensurable Case}

In the commensurable case, the following proposition shows that $\triangle_{\Sigma}^{\mu}$ satisfies most of the original postulates:

Proposition 3. $\triangle_{\Sigma}^{\mu}$ satisfies (IC0), (IC1), $\left(\right.$ IC2 $\left.^{*}\right),\left(\right.$ IC3 $\left.^{*}\right),($ IC5), (IC6), (IC6'), (IC7), (IC8) and (MAJ).

However, $\triangle_{\Sigma}^{\mu}$ falsifies $\left(\mathbf{I C 4}^{*}\right),(\mathbf{M I})$ and $(\mathbf{C S S})$. For the lack of space, we only provide a counter-example for $\left(\mathbf{I C} 4^{*}\right)$ :

Example 5. Let us consider $B_{1}=\{(\neg a, 2),(b, 1)\}, B_{2}=\{(a, 3),(b, 2)\}$, and $\mu=$ $a \vee b$. We have $B_{1}^{*} \models \mu$ and $B_{2}^{*} \models \mu$, and from Table $5, \operatorname{Mod}\left(\triangle_{\Sigma}^{\mu}(E)\right)=\left\{\omega_{3}\right\}$, and then $\triangle_{\Sigma}^{\mu}(E) \equiv a \wedge b$. Hence, on this example, $\triangle^{\mu}\left(\left\{B_{1}, B_{2}\right\}\right) \wedge B_{2}^{*}$ is consistent, but $\triangle^{\mu}\left(\left\{B_{1}, B_{2}\right\}\right) \wedge B_{1}^{*}$ is not.

\begin{tabular}{|c|c|c|c|c|c|}
\hline & $a b$ & $\kappa_{B_{1}}(\omega)$ & $\kappa_{B_{2}}(\omega)$ & $\nu_{E}(\omega)$ & $\Sigma\left(\nu_{E}(\omega)\right)$ \\
\hline$\overline{\omega_{0}}$ & $\begin{array}{lll}0 & 0\end{array}$ & 1 & 3 & $\langle 1,3\rangle$ & 4 \\
\hline$\omega_{1}$ & $\begin{array}{ll}0 & 1\end{array}$ & 0 & 3 & $\langle 0,3\rangle$ & 3 \\
\hline$\omega_{2}$ & 10 & 2 & 2 & $\langle 2,2\rangle$ & 4 \\
\hline$\omega_{3}$ & 11 & 2 & 0 & $\langle 2,0\rangle$ & 2 \\
\hline
\end{tabular}

Table 5. Profiles associated with interpretations

\subsection{The Incommensurable Case}

When belief bases are incommensurable, we obtain:

Proposition 4. $\boldsymbol{\Delta}_{\Sigma}^{\mu_{1}}\left(\right.$ E) satisfies $(\mathbf{I C 0}),(\mathbf{I C 1}),\left(\mathbf{I C 2}^{*}\right),\left(\right.$ IC3 $\left.^{*}\right),\left(\right.$ IC4 $\left.^{*}\right),($ IC5), (IC7) and (CSS).

However, $\mathbf{\Delta}_{\Sigma}^{\mu}$ falsifies (IC6), (IC6'), (IC8), and (MAJ). For the lack of space, we only provide a counter-example for (IC6).

Example 6. Let us consider $\mu=\top, E_{1}=\left\{B_{1}=\{(a, 1)\}, B_{2}=\{(\neg a, 1)\}\right\}$ and $E_{2}=\left\{B_{3}=\{(a, 1)\}\right\}$. From Tables 6 , we have $\boldsymbol{\Lambda}_{\Sigma}^{\mu}\left(E_{1}\right) \equiv \top$ and $\boldsymbol{\Lambda}_{\Sigma}^{\mu}\left(E_{2}\right) \equiv a$. Furthermore, we have $\boldsymbol{\Lambda}_{\Sigma}^{\mu}\left(E_{1} \sqcup E_{2}\right) \equiv \top$, but $\boldsymbol{\Lambda}_{\Sigma}^{\mu}\left(E_{1}\right) \wedge \boldsymbol{\Lambda}_{\Sigma}^{\mu}\left(E_{2}\right) \equiv a$. Hence, 
(IC6) is not satisfied since $\boldsymbol{\Lambda}_{\Sigma}^{\mu}\left(E_{1}\right) \wedge \boldsymbol{\Lambda}_{\Sigma}^{\mu}\left(E_{2}\right)$ is consistent, but $\boldsymbol{\Lambda}_{\Sigma}^{\mu}\left(E_{1} \sqcup E_{2}\right) \not=$ $\boldsymbol{\Lambda}_{\Sigma}^{\mu}\left(E_{1}\right) \wedge \boldsymbol{\Lambda}_{\Sigma}^{\mu}\left(E_{2}\right)$.

\begin{tabular}{|c|c|c|c|}
\hline $\mathrm{a} \mathrm{b}$ & $\nu_{E_{1}}(\omega)$ & $\nu_{E_{2}}(\omega)$ & $\nu_{E_{1}} \sqcup E_{2}($ \\
\hline $\begin{array}{l}\gamma_{0} \\
\end{array}$ & $\langle\mathbf{1}, \mathbf{0}\rangle$ & $\langle 1\rangle$ & \\
\hline 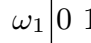 & $\langle\mathbf{1}$, & & \\
\hline$\omega_{2}$ & $\langle 0$ & & \\
\hline$\omega_{3} 11$ & $\langle\mathbf{0 , 1}\rangle$ & $\langle\mathbf{0}\rangle$ & $\langle 0,1,0\rangle$ \\
\hline
\end{tabular}

Table 6. Profiles associated with interpretations

Note that the non-satisfaction of (IC8) is due to the fact that when dealing with incommensurable belief bases, $\varangle_{\Sigma}^{E}$ is only a partial order. For instance, in [12], the fusion mode based on partial order does not satisfy (IC8).

Regarding the non satisfaction of the majority postulate, the situation is even worst. It can be shown that the Sum-based incommensurable belief base merging operator satisfies the majority independence postulate.

Proposition 5. $\mathbf{\Lambda}_{\Sigma}^{\mu}(E)$ satisfies (MI).

\section{$5 \quad$ Selection Functions of Compatible Scales}

This section restricts our merging operator to particular subsets of compatible scales, in order to derive more plausible conclusions. We discuss the following particular classes of compatible scales:

- bounded class $S^{(p)}$ : compatible scales such that the highest new rank assigned to a formula cannot exceed a fixed threshold $p$ (a positive integer).

- linear class $S^{l}$ : this class only proceeds to a linear transformation of initial ranks;

- weighted class $S^{w}$ : this class allows a proportional change of initial weights;

- shift class $S^{s}$ : this class allows to hold distance between ranks associated with two distinct formulas from a given source.

Table 7 gives formal definitions of these selection functions:

\begin{tabular}{c|c|c}
\hline Class & Notation & $\left\{\mathcal{S}\right.$ s.t. $\left.\forall B_{i} \in E, \forall \varphi \in B_{i}: \ldots\right\}$ \\
\hline \hline Bounded & $S^{(p)}$ & $\mathcal{S}(\varphi) \leq p$ \\
\hline Linear & $S^{l}$ & $\mathcal{S}(\varphi)=a_{i} . R_{B_{i}}(\varphi)+b_{i}$ with $a_{i}>0, b_{i} \geq 0$ \\
\hline Weighted & $S^{w}$ & $\mathcal{S}(\varphi)=a_{i} \cdot R_{B_{i}}(\varphi)$ with $a_{i}>0$ \\
\hline Shift & $S^{s}$ & $\mathcal{S}(\varphi)=R_{B_{i}}(\varphi)+b_{i}$ with $b_{i} \geq 0$ \\
\hline
\end{tabular}

Table 7. Particular classes of compatible scales

Bounded compatible scales offer a natural way to select a set of compatible scales, since in practice common scales are bounded. One can remark that the 
smallest possible $p$ is $p_{\min }=\max \left\{\left|B_{i}\right|: B_{i} \in E\right\}$, where $\left|B_{i}\right|$ represents the number of different rank (or ranks) in $B_{i}$. If $p<p_{\text {min }}$, then the set of compatible scales is empty.

The shift compatible scale class allows to hold distance relations between ranks of formulas. Indeed, when applying a such compatible scale $\mathcal{S}$, then: $\forall\left(\varphi_{i j}, R_{B_{i}}\left(\varphi_{i j}\right)\right),\left(\varphi_{i j^{\prime}}, R_{B_{i}}\left(\varphi_{i j^{\prime}}\right)\right) \in B_{i}, \mathcal{S}\left(\varphi_{i j}\right)-\mathcal{S}\left(\varphi_{i j^{\prime}}\right)=R_{B_{i}}\left(\varphi_{i j}\right)-R_{B_{i}}\left(\varphi_{i j^{\prime}}\right)$.

Weighted compatible scales are obtained by multiplying associated ranks $R_{B_{i}}\left(\varphi_{i j}\right)$ by a weight $a_{i}$. Intuitively, these weights may represent the reliability of sources (each $B_{i}$ has a reliability weight $a_{i}$ ), and the merging operator become a weighted sum. Linear compatible scales class generalizes the two above classes (weighted and shift).

We denote by $\boldsymbol{\varangle}_{\Sigma, S^{(p)}}^{\mu}\left(\right.$ resp. $\boldsymbol{\varangle}_{\Sigma, S^{l}}^{\mu}, \boldsymbol{\varangle}_{\Sigma, S^{w}}^{\mu}$, and $\left.\boldsymbol{\varangle}_{\Sigma, S^{s}}^{\mu}\right)$ the order obtained from Definition 5 by replacing $\mathbb{S}$ by $S^{(p)}\left(\operatorname{resp} . S^{l}, S^{w}\right.$, and $\left.S^{s}\right)$. Following subsections analyze the impact of restricting to particular classes on the cautiousness of our merging operator and on the satisfaction of rational postulates.

\subsection{Impact on Cautiousness}

As a first surprising result, restricting to classes of affine or linear compatible scales does not affect the result of merging:

Proposition 6. $\forall \omega, \omega^{\prime} \in \Omega, \omega \varangle_{\Sigma, S_{E}^{l}}^{E} \omega^{\prime}$ iff $\omega \mathbf{४}_{\Sigma, S_{E}^{w}}^{E} \omega^{\prime}$ iff $\omega \mathbf{४}_{\Sigma}^{E} \omega^{\prime}$.

However, inference based on bounded scales is in general more productive than $\boldsymbol{\Delta}_{\Sigma}^{\mu}$. In fact, inference from bounded scales depends on the value of $p$, and for a very particular value of $p$ the standard sum-based merging operator is recovered. Indeed, if all bases in $E$ have the same number of different ranks, equal to $p_{0}$, and that the maximal rank associated with formulas in each $B_{i}$ is $p_{0}$, then $\forall \omega, \omega^{\prime} \in \Omega: \omega \triangleleft_{\Sigma, S_{E}^{\left(p_{0}\right)}}^{E} \omega^{\prime}$ iff $\omega \triangleleft_{\Sigma}^{E^{\mathcal{S}_{p_{0}}}} \omega^{\prime}$.

Regarding inference based on shift compatible scales, it is also in general more productive than $\boldsymbol{\Lambda}_{\Sigma}^{\mu}$. In fact, we can even provide a criterion which allows to characterize the order on possible worlds induced by $\boldsymbol{\triangleleft}_{\Sigma, S_{E}^{s}}^{E}$.

Proposition 7. Let $\omega, \omega^{\prime}$ be two interpretations of $\Omega$. Then $\omega \boldsymbol{\varangle}_{\Sigma, S_{E}^{s}}^{E} \omega^{\prime}$ if and only if: i) $\Sigma\left(\nu_{E}(\omega)\right)<\Sigma\left(\nu_{E}\left(\omega^{\prime}\right)\right)$ and $\left.i i\right) \forall B_{i} \in\left\{B_{j} \in E, \omega^{\prime} \models B_{j}\right\}, \omega \models B_{i}$.

A full picture of the relationships between these different merging operators regarding prudence relations will be provided before the concluding discussion.

\subsection{Impact on Rational Postulates}

Table 8 summarizes the impact of selection functions on the satisfaction of postulates. In addition to Table $8, \boldsymbol{\Lambda}_{\Sigma, S^{(p)}}^{\mu}$ and $\boldsymbol{\Delta}_{\Sigma, S^{s}}^{\mu}$ both satisfy (IC0), (IC1), $\left(\right.$ IC2 $\left.^{*}\right),\left(\right.$ IC3 $\left.^{*}\right),($ IC5), and (IC7).

The main reason of the non-satisfaction of the majority postulate by $\boldsymbol{\Lambda}_{\Sigma}^{\mu}(E)$ is that new ranks which are assigned to belief bases by compatible scales are not 


\begin{tabular}{l||c|c|c|c|c|c}
\hline & IC4 $^{*}$ & (IC6) & (IC8) & (MAJ) & (MI) & (CSS) \\
\hline \hline$\triangle_{\Sigma}^{\mu}$ & - & $\sqrt{ }$ & $\sqrt{ }$ & $\sqrt{ }$ & - & - \\
\hline $\boldsymbol{\Delta}_{\Sigma}^{\mu}$ & $\sqrt{ }$ & - & - & - & $\sqrt{ }$ & $\sqrt{ }$ \\
\hline $\boldsymbol{\Delta}_{\Sigma, S^{s}}^{\mu}$ & $\sqrt{ }$ & - & - & - & - & $\sqrt{ }$ \\
\hline $\boldsymbol{\Delta}_{\Sigma, S^{(p)}}^{\mu}$ & $\sqrt{ }$ & - & - & $\sqrt{ }$ & - & - \\
\hline
\end{tabular}

Table 8. Rational postulates satisfied in commensurable and incommensurable cases

bounded. For instance, assume that $B_{1}$ contains $\varphi$ and $B_{2}$ contains $\neg \varphi$. Since compatible scales are not bounded, then even if $B_{1}$ is repeated $m$ times, it is always possible to find a compatible scale that assigns a high rank to formulas of $B_{2}$ (hence to $\neg \varphi$ ) which blocks the inference of $\varphi$. This explains why $\boldsymbol{\Delta}_{\Sigma, S^{(p)}}^{\mu}$ satisfies (MAJ) while other compatible based operators not.

$\triangle_{\Sigma}^{\mu}$ satisfies most of postulates except the fairness postulate (IC4 ${ }^{*}$ ) and (CSS). A natural question is whether there exists a single compatible scale that satisfies the fairness postulate and the consensus postulate. The following proposition provides a very particular case where $\left(\mathbf{I C 4}{ }^{*}\right)$ and $(\mathbf{C S S})$ hold.

Proposition 8. Let $E=\left\{B_{1},=\left\{\left(\varphi, R_{B_{1}}(\varphi)\right)\right\}, B_{2}=\left\{\left(\varphi^{\prime}, R_{B_{2}}\left(\varphi^{\prime}\right)\right)\right\}\right\}$. Let $\mathcal{S}$ be a compatible scale. Then: $\triangle_{\Sigma, \mathcal{S}}^{\mu}$ satisfies $\left(\mathbf{I C 4}{ }^{*}\right)$ and $(\mathbf{C S S})$ iff $\mathcal{S}(\varphi)=\mathcal{S}\left(\varphi^{\prime}\right)$.

However, in general, there is no hope to recover the satisfaction of the fairness and consensus postulates if one only uses a single compatible scale.

Proposition 9 (of impossibility). There is no single compatible scale such that $\triangle_{\Sigma, \mathcal{S}}^{\mu}$ satisfies the fairness and the consensus postulate for multi-set of sources $E$, namely: $\nexists \mathcal{S}$ s.t. $\forall E: \triangle_{\Sigma, \mathcal{S}}^{\mu}$ satisfies $\left(\mathbf{I C 4}{ }^{*}\right)$ or $(\mathbf{C S S})$.

For the counter example, it is enough to consider $E=\left\{B_{1}, B_{2}, B_{3}\right\}$ with $B_{1}=\{(a \wedge c, 1)\}, B_{2}=\{(\neg a, 1)\}$, and $B_{3}=\{(\neg c, 1),(a, 2)\}$.

\section{A comparative study}

This section provides a comparative study of our merging operators with respect to max-based merging and coherence-based merging. Let $\boldsymbol{\Lambda}_{M a x}^{\mu}(E)$ (resp. $\left.\triangle_{M a x}^{\mu}(E)\right)$ be defined exactly as $\boldsymbol{\Delta}_{\Sigma}^{\mu}(E)$ (resp. $\triangle_{\Sigma}^{\mu}(E)$ ) given by Definition 5 (resp. Definition 3), except that the sum operator $\Sigma$ is replaced by the maximum operator Max (see [5] for more details).

Note that in the commensurable case, the sum-based and the Max-based merging operators are incomparable. In the incommensurable case, we have a strict inclusion between these two inference relations, namely:

$$
\boldsymbol{\Delta}_{\Sigma}^{\mu}(E) \models \boldsymbol{\Delta}_{\operatorname{Max}}^{\mu}(E)
$$

Another way to deal with merging incommensurable bases is to view the set of bases to merge $E$ as a partially ordered belief bases $\left(K^{E},<_{K^{E}}\right)$ where $K^{E}$ is a multi-set containing all formulas in each bases of $E$, and $<_{K^{E}}$ is defined by: 
$\varphi_{i j}<_{K^{E}} \varphi_{i k}$ iff $\exists B_{i} \in E$ such as $\varphi_{i j} \in B_{i}, \varphi_{i k} \in B_{i}$ and $R_{B_{i}}\left(\varphi_{i j}\right)<R_{B_{i}}\left(\varphi_{i k}\right)$. Computing the result of merging comes down to select a set of preferred interpretation, according to $\left(K^{E},<_{K^{E}}\right)$. One way to define such preferred interpretations is to use the well-known set inclusion-based criterion defined by [13]:

Definition 6. An interpretation $\omega$ is said to be Incl-preferred to another interpretation $\omega^{\prime}$, denoted by $\omega \triangleleft_{E}^{I n c l} \omega^{\prime}$, iff: $\forall \varphi \in K^{E}$ s.t. $\omega \not \models \varphi$ and $\omega^{\prime} \models \varphi$, $\exists \psi \in K^{E}$ s.t. $\omega \models \psi$ and $\omega^{\prime} \mid \models \psi$ and: $\psi<_{K^{E}} \varphi$.

This leads to define a merging operator $\triangle_{\text {Incl }}^{\mu}$ based on $\triangleleft_{E}^{I n c l}$. Preferred beliefs for this merging operator are defined as follows: $\operatorname{Mod}\left(\triangle_{I n c l}^{\mu}(E)\right)=\operatorname{Min}\left(\operatorname{Mod}(\mu), \triangleleft_{E}^{I n c l}\right)$.

The following proposition expresses that when each belief base contains exactly one formula, namely $K^{E}$ is a set of propositional formulas, then $\triangle_{I n c l}^{\mu}$ and $\boldsymbol{\Delta}_{\Sigma}^{\mu}$ provide the same result:

Proposition 10. Assume that each $B_{i}$ exactly contains one propositional formula. Then $\forall \varphi \in \mathcal{L}, \quad \triangle_{\text {Incl }}^{\mu}(E) \models \varphi \quad$ iff $\quad \mathbf{\Delta}_{\Sigma}^{\mu}(E) \models \varphi$

Again, Proposition 10 shows that $\boldsymbol{\Lambda}_{\Sigma}^{\mu}$ has a different behavior in the incommensurable case, since if $B_{i}$ 's are commensurable and contains a single formula, then $\triangle_{\Sigma}^{\mu}$ is more productive than inclusion-based approach. Now, if $B_{i}$ 's contain more than one formula, then $\triangle_{\text {Incl }}^{\mu}(E)$ and $\triangle_{\Sigma}^{\mu}(E)$ are incomparable.

Example \%. Let $E_{1}=\left\{B_{1}, B_{2}, B_{3}\right\}$ be such that $B_{1}=\{(a \wedge b, 1)\}, B_{2}=\{(\neg a \wedge$ $b, 1)\}$ and $B_{3}=\{(a, 1)\}$. The sum-based operator will conclude $\{a\}$ whereas the inclusion-based merging operator will not. Now consider $E_{2}=\left\{B_{1}, B_{2}\right\}$ where $B_{1}=\{(b, 2),(a, 1)\}$ and $B_{2}=\{(\neg a, 2)\}$. Here, the inclusion-based merging operator will deduce $\{b\}$ whereas the sum-based merging operator will not.

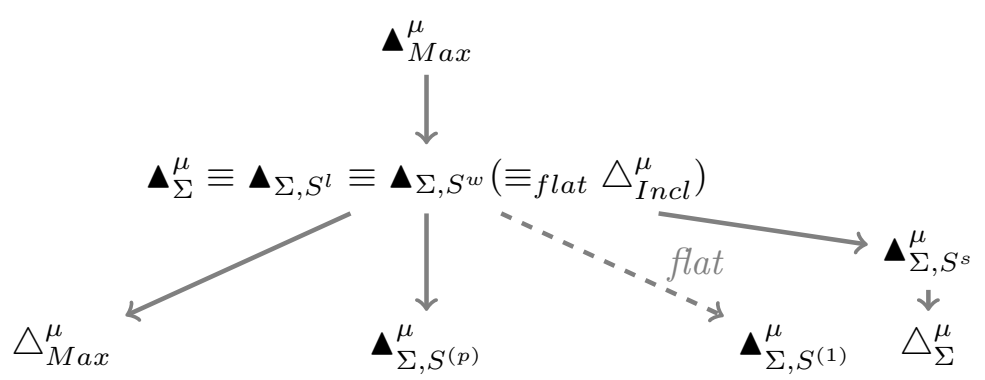

Fig. 1. Cautiousness of the different merging operators

Figure 1 summarizes the links between all operators in terms of cautiousness: $\triangle_{1} \rightarrow \triangle_{2}$ means that $\triangle_{1}$ can be inferred by $\triangle_{2}$. Flat case is also represented. In case of flat bases (namely each $B_{i}$ contains exactly one formula), then $\triangle_{\text {Incl }}^{\mu}$ is more cautious than if one uses bounded scales with $p=1$. In fact, when each belief base in $E$ contains a single formula, then we can check that the well-known cardinality-based inference can be recovered from bounded scales with $p=1$. 


\section{Conclusion}

This paper investigated the sum-based merging operator for incommensurable bases. We proposed a characterization of the merging result in terms of compatible scales and in terms of a Pareto-ordering. This paper showed that the behavior of the sum-based merging in incommensurable case departs from the commensurable case, regarding postulates satisfaction and cautiousness properties. In particular, the sum-based merging operator is no longer a majoritarian operator. We analyzed different classes of compatible scales. Bounded compatible scales allow to recover the majority operator, and some coherence based approaches [14] when bases contain a single formula. We also analyzed the fairness postulate $\left(\mathbf{I C} 4^{*}\right)$ and the new postulate proposed in this paper, called consensus postulate (CSS). We showed that there is no way to recover these postulates if a single compatible scale is selected. Lastly, this paper provided a comparative study between different merging operators discussed in this paper.

\section{References}

1. Everaere, P., Konieczny, S., Marquis, P.: Conflict-based merging operators. In: Proceedings of KR'08). (2008) 348-357

2. Lin, J.: Integration of weighted knowledge bases. Artificial Intelligence $\mathbf{8 3}(2)$ (1996) 363-378

3. Konieczny, S., Pino Pérez, R.: Merging information under constraints: a logical framework. Journal of Logic and Computation 12(5) (2002) 773-808

4. Guilin, Q., Liu, W., Bell, D.A.: Merging stratified knowledge bases under constraints. In: Proceedings of AAAI'06. (july 2006) 348-356

5. Benferhat, S., Lagrue, S., Rossit, J.: An egalitarist fusion of incommensurable ranked belief bases under constraints. In: Proceedings of AAAI'07. (2007) 367-372

6. Dubois, D., Lang, J., Prade, H.: Possibilistic logic. In: Handbook of Logic in Artificial Intelligence and Logic Programming. Volume 3., Oxford University Press (1994) 439-513

7. Spohn, W.: Ordinal conditional functions: a dynamic theory of epistemic state. Causation in Decision, Belief Change and Statistics (1988) 105-134

8. Williams, M.A.: Iterated theory base change: A computational model. In: Proceedings of IJCAI'95. (1995) 1541-1547

9. Meyer, T.: On the semantics of combination operations. Journal of Applied NonClassical Logics 11(1-2) (2001) 59-84

10. Nebel, B.: Belief revision and default reasoning: Syntax-based approaches. In: Proceedings of KR'91. (july 1991) 417-428

11. Liberatore, P., Schaerf, M.: Arbitration: A commutative operator for belief revision. In: Proceedings of the $2^{\text {nd }}$ World Conference on the Fundamentals of Artificial Intelligence. (1995) 217-228

12. Everaere, P., Konieczny, S., Marquis, P.: A diff-based merging operator. In: Proceedings of NMR'08. (2008) 19-25

13. Brewka, G.: Preferred subtheories: an extended logical framework for default reasoning. In: Proceedings of IJCAI'89. (1989) 1043-1048

14. Benferhat, S., Dubois, D., Prade, H.: Some Syntactic Approaches to the Handling of Inconsistent Knowledge Bases: a Comparative Study Part 2: the Prioritized Case. In Orłowska, E., ed.: Logic at Work: Essays Dedicated to the Memory of H. Rasiowa. Physica-Verlag (1999) 437-511 